\title{
Renaissance des études afromexicaines et production de nouvelles identités ethniques
}

Odile Hoffmann

\section{OpenEdition}

1 Journals

Édition électronique

URL : https://journals.openedition.org/jsa/3005

DOI : 10.4000/jsa.3005

ISSN : 1957-7842

Éditeur

Société des américanistes

Édition imprimée

Date de publication : 5 décembre 2005

Pagination : 123-152

ISSN : 0037-9174

\section{Référence électronique}

Odile Hoffmann, «Renaissance des études afromexicaines et production de nouvelles identités ethniques ", Journal de la Société des américanistes [En ligne], 91-2 | 2005, mis en ligne le 10 juin 2010, consulté le 03 septembre 2022. URL : http://journals.openedition.org/jsa/3005 ; DOI : https://doi.org/ 10.4000/jsa.3005 


\title{
RENAISSANCE DES ÉTUDES AFROMEXICAINES ET PRODUCTION DE NOUVELLES IDENTITÉS ETHNIQUES
}

\author{
Odile HOFFMANN *
}

Les travaux sur les populations noires au Mexique ont longtemps été cantonnés au cercle restreint de quelques spécialistes, principalement historiens. Depuis quelques années, les études se multiplient et abordent des thématiques contemporaines concernant ces populations certes minoritaires mais qui commencent à exprimer publiquement des revendications d'ordre culturel et, plus timidement, politique. La révision bibliographique permet de pointer les atouts et les faiblesses des courants théoriques actuels, tout en situant les enjeux de la problématique mexicaine sur la scène nationale et au sein des études " afro » en Amérique latine. On analyse ensuite les stratégies et moyens d'action des acteurs - universitaires, politiques, culturels - qui participent aux débats. Face à un phénomène d'ethnicisation rapide et largement exogène, une dernière partie explore quelques voies de recherche alternative. [Mots clés: identité noire, afrodescendants, afromexicain, Costa Chica, Mexique, ethnicisation.]

Renacimiento de los estudios afromexicanos y producción de nuevas identidades étnicas. Durante muchos años los estudios sobre poblaciones negras en México se enfocaron principalmente en temáticas históricas. Desde hace algunos años se empiezan a diversificar y multiplicar los estudios de corte más contemporáneo, respondiendo a una tímida pero real movilización de algunos grupos y personas negros, por ahora expresada en el campo cultural y, de manera menos frecuente, político. La revisión crítica de la bibliografia pasada y actual permite ubicar los retos y especificidades de la situación mexicana. Se analizan luego las estrategias y medios de acción de los actores que participan en los debates, principalmente académicos, políticos y activistas culturales. Frente a un proceso de etnicización rápido y en gran medida exógeno, se indagan finalmente algunas pistas alternativas de investigación. [Palabras claves : identidades negras, afrodescendientes, afromexicanos, Costa Chica, México, etnicización.]

The renaissance of Afromexican studies and the creation of new ethnic identities. For many years the studies on black populations in Mexico focused mainly on historic themes. During the past few years, studies regarding Mexican black populations have begun to multiply and to diversify towards a more contemporary approach. These

*IRD-CEMCA (Centro francés de estudios mexicanos y centroamericanos), Sierra Leona 330, Lomas de Chapultepec, 11000 Mexico D.F., Mexique [cemca.hoffmann@francia.org.mex].

Journal de la Société des Américanistes, 2005, 91-2, pp. 123-152. O Société des Américanistes. 
studies are responding to a small but real mobilization of some Afro-Mexican groups, which are venturing into the cultural and public arenas, and less frequently into the political realm. The critical revision of the present and past bibliography allows us to pinpoint the challenges and specificities of the Mexican situation. Furthermore, we analyze the strategies of the participants in the current debates, mainly academic, political and cultural activists. Facing a process of rapid and exogenous ethnicization, we are proposing alternative approaches and leads to consider in this field of study. [Key words : black identities, afrodescendents, afromexicans, Costa Chica, Mexico, ethnicization.]

Les travaux sur les populations noires mexicaines sont peu nombreux, mais se multiplient depuis quelques années, composant un champ d'études relativement peu exploré et problématique à plus d'un titre ${ }^{1}$. Une première série de questions se déduit du fait que la présence même de " populations noires » ou " groupes de population noire » ne fait pas consensus au Mexique. La plupart des habitants l'ignorent et certains spécialistes argumentent de sa disparition historique et achevée par métissage et « fusion » dans la société nationale (Velasco 2002), alors que d'autres au contraire la reconnaissent et intègrent « les afromexicains » dans la longue liste des " groupes ethniques » mexicains contemporains (Barabas et Bartolomé 1986).

Le débat ressemble à un dialogue de sourds tant les termes sont mal posés, les uns et les autres cherchant à reconnaître, pour des raisons opposées, un « groupe ethnique » constitué et délimité par des caractéristiques « culturelles » objectivement descriptibles et distinctes de celles « des autres », en général qualifiés de blancs ou d'indiens. On pourrait reconnaître là une tendance essentialiste, largement critiquée en anthropologie et qu'il est aisé de rejeter, tant il est vrai que les " traits culturels » s'empruntent, se transforment et ne prennent sens que dans des configurations historiques et socio-politiques données, sous l'effet d'actions et d'interactions de la part des acteurs sociaux mis en présence. Les auteurs cités eux-mêmes se réclament d'un modèle " processuel » et cherchent à rendre compte de «réalités » dans la mesure où le groupe assume comme siennes certaines représentations collectives spécifiques (ibid.), pouvant à l'occasion se réclamer d'un essentialisme stratégique nécessaire lors des négociations et mobilisations politico-ethniques.

Reste la question de savoir si, dans le contexte mexicain, il est pertinent de faire appel à une " identité noire " ou à une spécificité « noire " pour expliquer des processus sociaux plus généraux, pourquoi et, surtout, pour qui ? Comment, où et quand s'exprime éventuellement cette "identité », et que recouvre-t-elle ? L'approche instrumentaliste de l'identité (Glazer et Moynihan 1975) est insatisfaisante dans la mesure où, au Mexique, il n'existe pas de catégorisation externe institutionalisée qui reconnaisse "l'identité noire » et lui associe des mesures spécifiques ou des avantages matériels, politiques ou culturels. L'éventuelle 
expression ou revendication identitaire noire devrait donc se comprendre hors de toute stratégie identitaire directement liée à des politiques multiculturelles ou à des rapports de force entre « communautés » instituées.

En dehors des options radicalement essentalistes et instrumentalistes, il semble possible de proposer une voie médiane qui, clairement inscrite dans une optique constructiviste, récupère le vécu des premiers intéressés et permet de comprendre les processus de construction identitaire « de l'intérieur » - et pas seulement aux frontières - de l'ensemble formé par les personnes se réclamant d'une « différence » en tant que « noirs ${ }^{2}$ - ils sont rares - ou plus souvent " morenos» (« de couleur foncée »). On rejoint les préoccupations de Lévine (1999, p. 168) qui, tout en reconnaissant l'impact des catégorisations externes, cherche à réhabiliter une vision de l'intérieur de l'ethnicité. Il s'appuie sur une définition minimaliste de cette dernière comme étant « une méthode pour classer les gens (à la fois soi-même et les autres) qui se fonde principalement sur les origines - socialement construites »". L'acte de classer les gens selon leurs origines s'exerce tant aux frontières qu'au cœur même des groupes ainsi catalogués, c'est-à-dire qu'il ne se résume pas à élaborer les critères d'inclusion/exclusion propres à chaque groupe, mais qu'il intervient aussi dans la construction d'un consensus, interne au groupe, sur sa «culture ». Les deux processus sont également importants. Si l'anthropologie a récemment insisté sur le premier des termes, il nous faut aussi considérer les mécanismes de signification (filling-up ou « remplissage ») de ces catégories par les gens eux-mêmes (ibid., p. 171). Comme le note Sahlins, " que cela plaise ou non aux anthropologues, les gens - et pas seulement les dominants - réclament de la culture, et la réclament précisément dans sa modalité limitée, réifiée, essentialisée et atemporelle que la plupart d'entre nous rejetons désormais ${ }^{3}{ }^{3}$ (Sahlins 1999, p. 403. Traduction O. H.). Barth lui-même, à propos de son fameux article de 1969 (1995), précise bien qu'il n'est pas suffisant d'analyser les processus de frontières et que «des activités et institutions centrales et culturellement valorisées dans un groupe ethnique peuvent être profondément impliquées dans l'entretien des frontières en activant des processus internes de mise en cohésion " ${ }^{4}$ (Barth 1994, pp. 17-18. Traduction O. H.). L'ethnicité est donc un processus et un résultat de la catégorisation, celle-ci étant en permanence ré-élaborée en fonction des contextes et des espaces dans lesquels elle s'exerce. Si l'on suit ces quelques principes analytiques, le cas des populations afromexicaines est particulièrement intéressant puisque les processus de catégorisation sont en cours et ne sont pas encore cristallisés, contrairement par exemple à d'autres pays latinoaméricains, comme la Colombie, où l'existence d'un " groupe ethnique noir » fait désormais consensus (même si on peut et on doit en discuter les limites et le contenu).

Au Mexique, il faut commencer par se demander si le concept d'« ethnicité » est le mieux à même de rendre compte des dynamiques identitaires actuelles et quels sont les acteurs et les discours qui poussent dans ce sens. En effet, locale- 
ment, les populations afromexicaines semblent moins intéressées à définir leur " qualité ethnique » qu'à dénoncer la discrimination dont elles sont l'objet et à réclamer la reconnaissance de leur identité «mexicaine » qui est fréquemment mise en doute (Lewis 2000). La revendication de leur différence peut être interprétée en termes d'identité collective plutôt qu'ethnique. Comme le notait Wachtel (1992, p. 49) pour un tout autre contexte, celui des communautés andines, « on ne saurait voir de l'ethnicité partout » et il faut éviter les confusions qui, sous le terme " identité », traitent en fait de questions distinctes (voir également, sur ce thème de la confusion, Wieviorka 2004).

Le croisement de ces questions - identité ethnique ou collective, dimensions endogène et exogène de l'identité - ne peut se faire qu'en analysant les espaces où se forgent et s'exposent les processus de construction identitaire. L'espace est ici compris dans sa double acception : en tant qu'échelle ou niveau d'expression identitaire (individuelle, groupale ou collective), et en tant que lieu géographique concret autour duquel s'organise l'identification. Ce n'est pas la même chose que de se réclamer " noir » face au voisin ou face au chercheur, non plus de le faire au village ou dans la ville voisine de la même région, a fortiori dans la capitale. L'analyse des contextes - notamment institutionnels - de l'expression identitaire devra donc prendre en compte cette dimension spatiale, ce qui pourra nous aider à préciser, par exemple, les rapports entre identité collective et identité ethnique, ou encore entre identité et territoire.

Une seconde série de questions se rapporte à la façon dont sont menées les recherches sur ce thème aujourd'hui et comment elles se sont réalisées dans le passé. Une généalogie des travaux spécialisés dans ce domaine nous permettra de repérer les antécédents et les courants interprétatifs qui marquent encore les problématiques actuelles, d'en expliciter les faiblesses et les atouts et finalement de poser quelques pistes de recherche.

Ces deux séries d'interrogations se croisent évidemment. Je propose ici, à l'occasion de la révision de la littérature, d'aborder les questions théoriques et méthodologiques tout en gardant à l'esprit mon double objectif, à savoir : quelle place occupent aujourd'hui les études afromexicanistes au sein du débat intellectuel sur les populations noires, d'une part ? comment aborder l'analyse de ce " groupe social », d'autre part ? L'article se construit en trois parties. La première resitue les enjeux de la problématique afromexicaniste. La deuxième retrace la généalogie des travaux en signalant une forte tendance à l'essentialisation. Enfin, la troisième analyse les porteurs de l'ethnicisation en cours. En conclusion, j'explore quelques voies possibles de recherche.

Les interprétations avancées reposent sur deux terrains contrastés : l'un, dans la Costa Chica de Guerrero-Oaxaca (précisément les municipes de Marquelia et Cuajinicuilapa), est un travail ethnographique en cours depuis 2003 qui porte sur la distribution des ressources et sur les stratégies des acteurs pour se positionner dans le jeu politique local ${ }^{5}$; l'autre est un terrain « délocalisé » qui correspond à 
la sphère des interventions et expressions publiques liées à l'identification « noire » ou « afro » au Mexique. Ces deux approches sont complétées par le suivi et la révision des productions scientifiques nationales et étrangères sur ce sujet.

\section{Les populations noires au Mexique, le chaînon manquant ?}

La présence et l'importance des populations noires dans le sous-continent latino-américain sont désormais reconnues au-delà du cercle des spécialistes et font l'objet de vastes débats nationaux et internationaux, tant sur les scènes politiques que dans le domaine de la recherche. Les « afrodescendants 》- terme employé depuis la conférence internationale contre le racisme et la xénophobie à Durban, en 2001, par les organisations noires pour se différencier des afroaméricains des États-Unis - sont reconnus en tant que « communautés ethniques » par les textes constitutionnels dans plusieurs pays qui mettent en place des mesures spécifiques pour lutter contre la discrimination et promouvoir l'intégration dans la reconnaissance des particularités (par exemple en Colombie, voir Agudelo 1999).

Le Mexique participe à ces débats en tenant une position singulière. Si l'État mexicain a reconnu la nature pluriethnique et multiculturelle du pays (réforme constitutionnelle de 1992), aucune loi d'application ne s'en est suivi. Les options politiques du pays, affirmées dans les années 1990 (adhésion à l'Organisation de coopération et de développement économique ou OCDE, Accord de libre échange avec le Canada et les États-Unis), lui permettent difficilement d'élaborer des lois au bénéfice de groupes spécifiques - principalement les indiens ${ }^{6}-$, qui contreviendraient aux principes de libéralisation de la circulation des biens et des services (Hoffmann 2001). Malgré les mobilisations politiques, les demandes sociales, les protestations et l'insurrection néo-zapatiste de 1994, les ambiguittés politiques ne sont pas levées et le multiculturalisme affiché au niveau fédéral ne se traduit par aucune mesure concrète.

Concernant les populations noires ou "d'origine africaine ", le Mexique est encore plus ambigu. Si l'ampleur de l'esclavage et de la présence noire est un fait historique désormais avéré et bien documenté pour la plupart des régions du pays, il n'en va pas de même pour les populations noires contemporaines dont l'existence en tant qu'individus, et plus encore en tant que groupe social, n'est reconnue par aucun texte légal. Pour autant, des institutions culturelles, certains chercheurs et quelques militants de la cause noire contribuent à construire, à partir de points de vue qui ne concordent pas forcément, un nouveau champ d'études ou d'interprétations sur les populations noires au Mexique. Je voudrais ici explorer les voies de cette construction intellectuelle et politique récente, de cette "renaissance » des études afromexicanistes ${ }^{7}$, en la replaçant dans son contexte institutionnel, politique et social.

Quelques hypothèses fortes guideront l'analyse. La première est à vrai dire déjà amplement confirmée et consiste à poser qu'au Mexique le spectre identitaire 
est entièrement occupé par l'identité nationale, d'une part, par les identités indiennes, d'autre part ${ }^{8}$. La première symbolise l'unité du pays alors que les secondes légitiment et organisent la diversité culturelle, la cohérence des deux options étant assurée par le discours du métissage, déjà ancien (depuis la fin du $\mathrm{XIX}^{\mathrm{e}}$ siècle) et renouvelé après la Révolution de 1910-1920. L'émergence d'autres revendications identitaires ne saurait dans ce contexte relever que de l'exception ou de l'exotisme, et pour des populations bien particulières par leur origine (les Chinois arrivés des Philippines au XvII ${ }^{\mathrm{e}}$ siècle, les Japonais avec le travail forcé, plus récemment les Coréens, etc.). Les études sur les populations noires se sont longtemps cantonnées à ce schéma, faisant « du noir » un être historiquement situé - et disparu.

Avec le regain d'intérêt pour les populations noires contemporaines se pose la question du cadre conceptuel à adopter; et c'est là qu'intervient ma seconde hypothèse : le développement historique de la population noire fait du Mexique un cas spécifique en Amérique latine, pour lequel les interprétations les plus contemporaines ne s'appliquent pas, ou difficilement. Ce qui expliquerait en partie la relative pauvreté théorique des recherches en ce domaine, mais pourrait se transformer en un puissant stimulant si le dynamisme des études se poursuit et s'accroît.

Contrairement à d'autres pays d'Amérique latine, en effet, il n'existe pas au Mexique de mouvement social de revendication identitaire noire qui pourrait justifier des approches analytiques en termes de mouvement social et politique (Touraine 1988). La population concernée est numériquement très minoritaire (quelques dizaines de milliers pour 100 millions d'habitants au Mexique en 2000, nous reviendrons plus loin sur ces chiffres) et politiquement inexistante en tant que telle. Il n'y a pas d'affichage de pratiques culturelles ou religieuses qui relèveraient d'une "identité afro " mobilisable pour des enjeux politiques, comme il peut y en avoir au Brésil ou à Cuba (Argyriadis et Capone 2004). Il n'existe pas non plus - sauf exceptions mentionnées plus loin - de mesures spécifiques autour desquelles s'organiseraient les revendications de groupes ou de collectifs « noirs », ce qui rend diffỉcile les interprétations désormais classiques en matière de construction et d'instrumentalisation identitaires : il n'y a objectivement aucun intérêt - ni politique, ni idéologique ou matériel - à « être » (devenir; se dire) noir. Si construction il y a, elle viendra d'ailleurs. Ni le discours officiel ni les acteurs politiques influents sur le plan national n'utilisent de catégorisation particulière pour se référer aux populations qui s'auto-définissent comme « morenas » ou « afrométisses ».

Pour leur part, les réflexions post-modernes sur l'invention identitaire et la capacité des individus à gérer leurs identités multiples de façon relationnelle et situationnelle (Hall 1994) se heurtent au fait que celles-ci ne peuvent s'exprimer que s'il existe des cadres légitimants, ce qui précisément fait défaut aux « noirs » mexicains qui n'occupent aucune place sur l'échiquier identitaire national. Sans 
dialogue possible avec un « autre » qui leur reconnaîtrait leur propre altérité, et en tout cas pas avec l'État, les afromexicains n'ont pas de frontière à traverser pour s'intégrer éventuellement dans les autres catégories identitaires disponibles (indiens, métis, blancs). Ils restent dans un entre-deux qu'ils assument en tant qu' " afrométis » ou "morenos », et le plus souvent en tant que "mexicains » (Lewis 2000), c'est-à-dire, dans les deux cas, en dehors des catégorisations ethniques en vigueur.

Cette option reste toutefois ouverte aux deux extrêmes de l'espace de vie, dans des contextes où « être noir » peut devenir pertinent et participer aux dynamiques sociales. Au niveau local, celui où les différences se gèrent au quotidien avec ou sans appareil conceptuel explicite, les « morenos » font l'expérience du racisme et de la discrimination à travers des paroles et des actes, les plus anodins comme les plus élaborés, de la part de l'ensemble de leurs voisins non noirs (Castillo 2000). Cette expérience de racisme partagé est le plus sûr ciment d'une "identité » - dans le sens d'un vécu identique -, tout du moins d'une altérité sans cesse rappelée par leurs interlocuteurs ${ }^{9}$. Elle peut alors donner lieu à toutes sortes de stratégies individuelles pour fuir le stigmate (négation), le renverser (affirmation) ou l'ignorer (l'évitement). Les approches développées par Goffman (1991) en terme d'interaction, utilisées notamment par Cunin (2004) en Colombie dans des contextes similaires, peuvent nous aider à comprendre les ambiguïtés et contradictions qui caractérisent souvent les positionnements identitaires des afrométis. Ambiguïtés qui interdisent de parler d'une identité évidente ou « naturelle », mais qui, cependant, mettent toujours en jeu la dimension « raciale » de la différence.

À l'autre extrême de l'espace social, les arènes internationales proposent à leur tour des catégories opérationnelles pour penser l'identité noire. Les réseaux de militantisme afro, les agences internationales spécialisées contre le racisme ou les textes de l'Organisation des Nations Unies sur l'esclavage sont autant de sources qui fournissent des instruments légitimes de catégorisations inexistants au niveau national. L'une des conceptualisations récentes est celle de la diaspora, concept dont l'usage serait légitimé par la dé-territorialisation originelle traumatique et la dispersion postérieure des personnes esclavagisées aux quatre coins du monde et, principalement, en Amérique. La question ne fait pourtant pas consensus. Quels sont les mythes communs qui donneraient cohérence à une éventuelle " diaspora noire »? Quels en sont les instruments (rituels par exemple) et les moyens d'expression? Sans rentrer dans la polémique, disons simplement que la plupart des sociétés noires ou afrométisses américaines ne partagent pas cette arène globalisée, mais s'ancrent au contraire dans des réalités extrêmement localisées, territorialisées, aux prises avec des problèmes d'alliances et de rivalités qui conditionnent leur survie matérielle et spirituelle. Pourtant, la voie reste ouverte pour une poignée d'activistes qui, bien que minoritaires, orientent singulièrement les représentations collectives en participant aux débats et aux mobilisations internationales. Dans l'immédiat, la notion de diaspora reste largement 
étrangère aux populations afromexicaines et offre difficilement un cadre théorique opérationnel.

Par ce rapide survol de la situation contemporaine des afromexicains, on a posé quelques jalons utiles pour la suite de l'argumentation : le Mexique apparaît comme le "chaînon manquant » en Amérique latine. Le terme vient d'un activiste noir qui exprimait ainsi le fait que, selon lui, la spécificité du Mexique (absence de catégorisation consensuelle - endo- ou exogène - et de mouvement de revendication ethnique noir) l'excluait de fait de l'agenda collectif assumé par les réseaux afro internationaux. Toutefois, aux niveaux micro et macro, on peut repérer des sources d'identification « noire » éventuellement mobilisables pour une ethnogénèse qui s'annonce. S'il est aujourd'hui aventureux de parler d'une " ethnicité noire » au Mexique, on ne peut nier l'existence d'une altérité vécue et d'expressions d'identités collectives assumées par les « afrométis ».

Les particularités de la situation mexicaine contemporaine découlent dans une large mesure de l'histoire du peuplement noir. Comme partout en Amérique latine, les populations noires sont descendantes d'individus arrivés au Mexique en tant qu'esclaves. Ils étaient employés dans de nombreux secteurs, parfois concentrés par région (mines, plantations de canne à sucre, élevage), mais le plus souvent dispersés autant à la campagne qu'en ville (artisanat, emplois domestiques, ouvriers), dans pratiquement toutes les régions du pays (Martínez Montiel 1994). Durant la période coloniale, ils connaissent l'histoire classique et dramatique de résistances, révoltes, fuites et constructions de palenques ${ }^{10}$, particulièrement dans les régions de plantations comme le Veracruz, où l'esclavage persiste jusqu'au XIX ${ }^{\mathrm{e}}$ siècle (Naveda 1987; Carroll 1991). Ailleurs, dès le début du $\mathrm{XVIII}^{\mathrm{e}}$ siècle et grâce à la récupération démographique indienne, les arrivées d'esclaves diminuent (Aguirre Beltrán 1972, p. 85) et le métissage s'accentue, principalement mais pas exclusivement, avec les indien(ne)s avec lesquel(le)s ils partagent la condition de subordonnés face aux Espagnols, créoles et métis. L'arrêt « précoce » d'importation d'esclaves noirs a eu pour conséquences principales l'intensification du métissage et la diminution rapide du pourcentage d'esclaves parmi la population noire, deux traits qui marquent la spécificité des populations noires du Mexique par rapport à celles d'autres pays d'Amérique latine ${ }^{11}$. Au moment de l'abolition de l'esclavage (interdit une première fois en 1810, aboli en 1817 mais avec le dernier décret d'abolition signé par Vicente Guerrero en $1829^{12}$ ), les populations noires (negros, pardos y mulatos dans les recensements) du Mexique sont déjà largement métissées et composées de paysans, ouvriers et artisans « libres » (comme pouvaient l'être les classes pauvres du $\mathrm{XVIII}^{\mathrm{e}}$ siècle, le plus souvent soumises à des mécanismes féroces de domination caciquiste, clientéliste ou paternaliste) ${ }^{13}$.

Mais les phénomènes sont inégalement répartis et donnent lieu à des dispositifs socio-géographiques particuliers. Dans certaines régions où le métissage est tardif et la proportion de noirs plus élevée, des identités régionales particulières 
intègrent et rappellent les origines noires sans s'y réduire : jarochos au Veracruz, guaches dans les terres chaudes de Morelia, mascogos au Coahuila. Ailleurs la fréquence de phénotypes noirs (couleur de peau, type de cheveux) peut être élevée sans avoir (pour l'instant?) donné naissance à des constructions identitaires particulières. La seule exception concerne sans doute la Costa Chica (côte pacifique de Guerrero-Oaxaca), région «multiethnique » où cohabitent groupes indiens, morenos et métis qui s'auto-identifient comme tels ${ }^{14}$ (Pépin Lehalleur 2003 ; Campos 1999 ; Neff 1986). La population totale s'élève à plusieurs dizaines de milliers de personnes (à la différence des indiens, il n'existe aucun recensement spécifique pour les populations noires). C'est dans cette région que s'expriment - et s'étudient - les principales revendications identitaires noires actuellement au Mexique. C'est également celle où s'élabora la première étude ethnographique sur les populations noires au Mexique (Aguirre Beltrán 1989 [1958]).

\section{LE DISPOSITIF SCIENTIFIQUE ET INSTITUTIONNEL}

\section{Généalogie}

L'initiateur des études afromexicanistes est sans contexte Gonzalo Aguirre Beltrán. Médecin d'origine, Aguirre Beltrán est déjà anthropologue lorsque Manuel Gamio, chef du département de démographie au ministère de l'Intérieur, lui demande une étude sur la population noire au Mexique, en 1942. S'ensuit un remarquable travail historique qui, pour la première fois, retrace en détail, sources d'archives à l'appui, les origines et l'ampleur du trafic esclavagiste, les rythmes d'arrivée et la répartition des esclaves, les secteurs d'activités et les processus de métissage, concluant sur l'importance de l'apport des populations d'origine africaine à la culture et à l'histoire mexicaines (Aguirre Beltrán 1972 [1946]). Quelques années plus tard, et après un séjour aux États-Unis où il étudie avec Herskovits, Gonzalo Aguirre Beltrán réalise la première étude ethnographique, dans le village de Cuajinicuilapa sur la côte de Guerrero (publiée en 1958). Â l'époque encore isolée géographiquement (la route panaméricaine n'est construite que dans les années 1960) et habitée principalement de populations peu métissées, la Costa Chica apparaît comme l'ultime témoignage d'une population noire en voie de disparition par métissage. Si Aguirre Beltrán parle de « noirs » et forge la notion « d'afro-métis » à ce propos ${ }^{15}$, c'est pour mieux en souligner le caractère exceptionnel et réaffirmer sa thèse de l'intégration des « noirs et leurs mélanges » (negros y sus mezclas) à la société nationale mexicaine ${ }^{16}$.

L'intégration se fonderait historiquement sur deux processus qui différencient les noirs des autres groupes subordonnés, en l'occurrence les indiens. D'une part, les traits culturels des noirs ne seraient pas suffisamment distinctifs pour servir d'instrument d'identification ethnique ; les traits raciaux auraient pour leur part rapidement disparu par métissage répété. À la fin de la période coloniale, il n'était 
pas possible d'asseoir des discriminations fortes (incapacidades asignadas) sur ces différences ténues. En contrecoup, il n'existait pas de support solide à la constitution ou au maintien de groupes séparés pour les populations noires et mulâtres (Aguirre Beltrán 1972 [1946], p. 287). D’autre part, dans la société coloniale, le système de castes assignait une place subordonnée mais reconnue aux indiens, selon un dispositif de ségrégation qui, même s'il fut théorique, idéologique et jamais totalement accompli dans la pratique sociale, persista jusqu'aux premiers temps de la société nationale indépendante. Ce n'est qu'à la fin du $\mathrm{XIX}^{\mathrm{c}}$ siècle que les réformes libérales abolirent les " privilèges » et droits spécifiques reconnus jusqu'alors aux communautés indiennes, notamment les droits sur la terre. Au contraire, la population afrométisse et métisse, sans place reconnue par le système colonial, deviendra la base du nouveau système indépendant qui prétend s'appuyer sur une population " nationale » et a besoin de cette masse, antérieurement marginalisée, mais non constituée en caste clairement séparée, pour se construire (ibid., p. 291). Ainsi, des mécanismes politicostructurels - le besoin d'intégrer les couches populaires pour faire exister la " société nationale » post-coloniale - et socio-culturels - le mélange de traits raciaux et culturels - auraient convergé vers une intégration presque complète des populations noires et mulâtres dans la société nationale et vers leur conséquente disparition comme groupe spécifique au sein de la société contemporaine. Notons que, dans ses analyses, Aguirre Beltrán soutient que les différences ethniques se créent et se transforment dans des relations de pouvoir; pour les noirs comme pour les indiens, dans une approche anti-essentialiste avant l'heure qui cependant ne fait pas d'émules et restera longtemps inaperçue.

La thèse de l'intégration a eu un tel succès qu'elle bloqua pour longtemps toute étude sur les populations noires contemporaines, jugées "peu authentiques » et de toutes façons vouées à disparaître rapidement. Il faut dire qu'à la même époque (années 1940-1960) l'anthropologie mexicaine se concentre sur l'étude des groupes indiens sous le double impact de la politique publique indigéniste ${ }^{17}$ et du développement théorique et méthodologique dû à plusieurs chercheurs mexicains et étrangers qui fondent la réflexion anthropologique nationale à partir d'études de cas pris en régions indiennes (Aguirre Beltrán, prologue à l'édition 1972 de son ouvrage La población negra en México...).

Ce déficit de légitimité, qui grève jusqu'à aujourd'hui l'ethnographie afromexicaniste, affecte moins le champ de la recherche historique qui continue à se développer. Elle trouve des relais et se poursuit aujourd'hui dans plusieurs universités de province ${ }^{18}$, au Colegio de México ${ }^{19}$, à l'institut Mora ${ }^{20}$ et notamment à l'Instituto Nacional de Antropología e Historia (INAH) qui vient de lancer une collection « Africanía » dont le premier ouvrage reflète les travaux du séminaire sur les « Populations d'origine africaine » (Velázquez et Correa 2005). Les approches historiques précisent les conditions régionales de l'esclavage (Palmer Colin 1993 ; Naveda Chávez-Hita 1987; Chávez Carvajal 1995 ; Guevara 
Sanginés 1994 ; Herrera Casasús 1994), mais s'intéressent également aux modes de vie de certains groupes (les femmes noires à Mexico, Velázquez Gutiérez 1994), aux croyances (travaux sur l'inquisition : Alberro 1988; Castañon González 2002) et en général à l'apport de la "culture afroaméricaine» à la culture nationale (Martínez Montiel 1993a ; 1993b ; 1994) ${ }^{21}$. En tout état de cause, l'intérêt d'une approche historique des populations noires ne s'est jamais démenti, pas plus que celle des études du « folklore » (musique, danse, tradition orale. Voir Gutiérrez Ávila 1988) qui trouve en Gabriel Moedano Navarro (1997) son plus illustre spécialiste.

En anthropologie, cependant, le travail pionnier de Gonzalo Aguirre Beltrán ne fera pas école sur ce thème, alors que lui-même assume, à la direction de l'Institut national indigéniste et, plus tard, du CIESAS (Centro de Investigaciones y Estudios Superiores en Antropología Social), un rôle de premier plan dans le développement de l'anthropologie mexicaine. Ce n'est que dans les années 1980 qu'un autre anthropologue de renom, Guillermo Bonfil Batalla, donne une nouvelle impulsion à la recherche ethnographique en créant le programme « $\mathrm{La}$ troisième racine » au sein de la Dirección General de Culturas Populares (DGCP, aujourd'hui inscrite au Consejo Nacional para la Cultura y las Artes, CONACULTA). Dirigé par Luz María Martínez Montiel (1993b, ouvrage coordonné par Bonfil), le programme vise à reconnaître l'apport (dans la danse, la musique, la cuisine, la littérature orale) des populations d'origine africaine à la culture nationale et donne lieu à de nombreuses recherches ponctuelles et publications sur ces thèmes, à des rencontres nationales et internationales et finalement à des débats parfois novateurs. Il légitime l'approche ethnographique dans les sphères scientifiques et institutionnelles et, surtout, il insère ce champ de recherches dans un cadre international. Le Mexique participe ainsi au programme de l'UNESCO intitulé « La route de l'esclave » qui a promu pendant une dizaine d'années des rencontres et publications conjointes entre pays d'Afrique et d'Amérique latine. Les représentants étaient, pour le Mexique, Luz María Martínez Montiel et, pour la Colombie, Jaime Arocha, tous deux anthropologues qui s'occupent, dans leurs pays respectifs, du renouveau des études afroaméricanistes contemporaines et soutiennent une approche en termes de " racines africaines » des cultures noires latinoaméricaines.

Il n'est désormais plus possible d'ignorer la production scientifique spécialisée. Même si tous les articles commencent infailliblement par se plaindre du manque d'antécédents, ceux-ci existent bel et bien. Certes ils sont diffíciles d'accès, souvent très partiels dans leurs approches, parfois mal documentés et de toutes façons beaucoup moins nombreux que ceux consacrés aux mondes indiens, mais ils constituent un corpus non négligeable. Une première révision bibliographique de Moedano Navarro (1992) et un travail en cours de Cristina Díaz Pérez (1994, à partir de son mémoire de licence) recensent quelque mille titres ; les mémoires de maîtrise et les doctorats en anthropologie se multi- 
plient, témoignant d'un réel intérêt des étudiants et de leurs professeurs pour ce sujet.

Les années 1990 marquent un véritable palier dans l'évolution des études afromexicanistes, jusque-là pratiquement cantonnées à la recherche historique et culturelle. Mais les approches ethnographiques actuelles présentent encore des faiblesses qui s'expliquent en partie par leur histoire.

\section{La tentation afrogénétique et l'impossible définition}

Le courant afromexicaniste n'a pour l'instant pas réussi à se dégager du carcan nominaliste qui veut que l'on précise d'abord « de qui parle-t-on ? » en termes d'appartenance ethnique. Barabas et Bartolomé (1986), dans la seconde édition de leur ouvrage de synthèse Etnicidad y pluralismo cultural. La dinámica étnica en Oaxaca, incluent les « afromexicains » dans les « 17 groupes ethniques de Oaxaca ». Cette position s'explique notamment par le fait que l'État de Oaxaca dispose d'une législation qui reconnaît, institue et régule la différence ethnique. Dans le domaine électoral notamment, les textes valident les formes d'élection des maires selon les « us et coutumes», qui peuvent varier d'un municipe à l'autre (à main levée, désignation par le conseil des anciens, avec ou sans participation des femmes et des « étrangers » au village, etc., voir Recondo 2001). Le « dispositif ethnique » déjà construit et reconnu par les institutions est donc là plus facile à transposer vers les populations noires. Pourtant, et contrairement aux groupes indiens voisins, il n'existe pas d'instrument d'estimation et de description statistique du « groupe ethnique afrométis ». Les indicateurs classiques au Mexique ne fonctionnent pas (la langue, la tenue vestimentaire, l'organisation sociale "traditionnelle») et chaque auteur élabore comme il peut ses propres discriminants, construits le plus souvent sur le sens commun propre à la situation qu'il étudie. Le chercheur en vient à adopter les catégories d'usage de ses interlocuteurs, le plus souvent sans critique préalable approfondie, avec le risque de reproduire et de transmettre les stéréotypes de la différence - ou de l'indifférence. C'est ainsi que « le noir » s'affronte violemment à l'indien dans la Costa Chica (Flanet 1977), une vision récurrente ${ }^{22}$ - parmi d'autres - jusqu'à aujourd'hui et qui, par l'usage du singulier notamment, exprime bien la nature stéréotypée de ces représentations.

En fait, il n'existe aucun consensus sur la définition des afrométis en tant que " groupe ethnique », encore moins sur ses éventuelles frontières. Celles-ci sont constamment renégociées selon les contextes d'altérité et diffèrent d'une localité à l'autre, d'une période à l'autre, en fonction des alliances et des tensions politiques, économiques ou sociales du moment. Le modèle barthien est efficace pour repérer ces mouvements de frontières, mais il est inopérant dans une optique de « définition » de groupe ethnique, pour lequel il n'a pas été pensé (Barth 1995 [1969]). Pour sa part, le modèle d'interprétation en termes de « relations interethniques » ne peut fonctionner - difficilement - que sur la base des monographies 
qui, seules, permettent de décrire comment localement se génèrent et s'interprètent les différences : à tel endroit entre noirs et indiens, à tel autre entre blancs et afro-indiens, à tel autre encore entre les trois groupes en présence, etc. (Cervántes Delgado 1984). Mais, on l'aura compris, la validité d'une telle approche diminue en proportion directe de la prétention à la généralisation ou à la théorisation. Comment en effet concevoir « l'interethnique » sans d'abord isoler des " groupes ethniques », chose qui précisément est encore - malheureusement - au cœur des débats et des polémiques ? C'est là sans doute que s'expose le plus clairement l'impasse théorique qui bloque nombre de chercheurs : tant qu'elle cherchera à définir les limites d'un éventuel « groupe afrométis » (ou afromexicain), l'anthropologie mexicaine sera incapable de saisir les processus de construction et déconstruction permanentes de cette entité sociale volatile, incertaine et pourtant active dans le champ social.

L'approche ethnographique qui pourrait éviter ce biais et « se décentrer » de la problématique ethnicisante s'y plonge parfois sans retenue. Il faut dire qu'elle est le plus souvent le fait d'étudiants, débutant en anthropologie, qui investissent le temps d'observation nécessaire et rendent compte de leurs travaux dans des mémoires de licence ou maîtrise plus ou moins bien documentés et rarement publiés. La plupart se réfèrent aux travaux de Aguirre Beltrán (1989) dont les descriptions ethnographiques (réalisées, rappelons-le, il y a plus d'un demi-siècle) soulignaient les traces d'africanité dans la façon de marcher, de construire sa maison ou de porter les enfants par exemple, mais aussi dans certains rites de guérison et croyances religieuses. L'influence du maître est d'autant plus grande qu'il y a peu de contre-poids, les étudiants mexicains - et leurs professeurs connaissant mal la littérature internationale sur les populations noires contemporaines. Mais, surtout, les descriptions d'aujourd'hui sont décontextualisées (Martínez Maranto 1994 ; Cruz 1989), alors qu'Aguirre Beltrán (1972 ; 1989) prônait une vision politique de la différence, clairement inscrite dans des relations de domination qu'il analysait.

L'intérêt de cette ligne de travail est cependant d'avoir apporté de la documentation actualisée. Ses limites se trouvent rapidement au niveau théorique, avec un double " dérapage » : au moment de qualifier certaines pratiques de " noires », voire d'" africaines », et en assimilant des pratiques, ou des ensembles de pratiques, à une identité collective arbitrairement nommée « noire » par le chercheur.

Dans le premier cas, la qualification d'une pratique donnée renvoie au registre de l'authentification, par le spécialiste qu'est l'ethnologue, d'une origine supposée « africaine » qui relègue au second plan les influences européennes ou mésoaméricaines, selon un mécanisme d'exclusion qui semblerait ignorer les brassages culturels particulièrement intenses depuis plusieurs siècles. La construction intellectuelle qui fonde ces interprétations « afrogénétiques » (Arocha 1999) prétend combler des décennies et siècles de méconnaissance des "spécificités 》 noires, interprétée comme racisme institutionnel et social contre lequel il faudrait lutter. 
Elle provient surtout, en grande partie, de la diffīiculté à penser le métissage autrement que comme négation des cultures " originelles », ce qui amène, en contrecoup et par facilité, à ne légitimer que "les origines » au détriment d'une conceptualisation plus ouverte, dialectique et dynamique des interactions culturelles (Gruzinski 1999). Cette diffículté doit évidemment être mise en relation avec l'histoire de la pensée du métissage au Mexique qui, depuis le XIX ${ }^{\mathrm{e}}$ jusqu'au début du $\mathrm{xx}^{\mathrm{e}}$ siècle, voyait dans "la race cosmique" (Vasconcelos 1958) l'homme du futur, délivré de ses atavismes culturels considérés comme obstacles au développement et à la construction nationale. Relayée par l'idéologie postrévolutionnaire puis l'indigénisme ofliciel qui prônait l'assimilation et l'intégration des indiens, désormais critiquée pour son eurocentrisme et son racisme latent, cette conception du métissage n'a pas été actualisée en tant que telle. Elle a plutôt été substituée à la fin du $\mathrm{xx}^{\mathrm{e}}$ siècle par une vision ethnicisante des rapports socio-politiques, elle-même liée, d'une part, à la reconnaissance du caractère multiculturel et pluriethnique de la nation, d'autre part, aux mobilisations sociales et politiques de ces dernières décennies. Les groupes indiens, notamment, revendiquent désormais leur accès aux droits et à la participation citoyenne sur la base de leurs identités ethniques. La conceptualisation de la situation afromexicaine se calque ainsi sur un modèle construit dans d'autres sphères.

Une exception importante à cette tendance particulariste et excluante concerne la région du sud de l'État de Veracruz, conceptualisée comme "la Caraïbe afro-andalouse » par García de León $(1992 ; 1993)$ qui met ainsi en lumière l'inextricable mélange des influences indiennes, caribéennes (notamment cubaines à la fin du XIx ${ }^{\mathrm{e}}$ et au début du $\mathrm{Xx}^{\mathrm{c}}$ siècle), de l'apport africain et celui des colons européens d'origine espagnole. Comme le montrent bien les travaux de quelques universitaires (Alcántara López 2002), la musique, la danse, la gastronomie et d'autres expressions culturelles rendent compte de cet héritage complexe dont ne peut s'approprier aucun des lointains prédécesseurs en particulier. Loin des schémas réducteurs de l'interprétation afrogénétique, ces chercheurs mettent en avant la créativité culturelle et sociale de ces sociétés régionales qui ne s'affichent nullement comme "noires» tout en revendiquant des influences nettement africaines (voir Figure 1).

Le deuxième dérapage théorique concerne le passage du singulier au collectif, l'assimilation d'éléments diversifiés à un ensemble construit et signifiant sur le plan identitaire. Dans le Sud du Veracruz, la Costa Chica ou les terres chaudes du Balsas, nul ne peut nier l'origine africaine de tel ou tel trait, allié à tel autre nettement mésoaméricain ou européen et dont l'arrangement est globalement distinct de celui des voisins. Tout un chacun peut également constater la présence de phénotypes nettement « noirs » ou de pratiques gestuelles, que ce soit dans la danse ou le quotidien, qui marquent la différence avec les régions indiennes et métisses de l'altiplano par exemple. Pour autant, jusqu'à présent, il n'est pas 


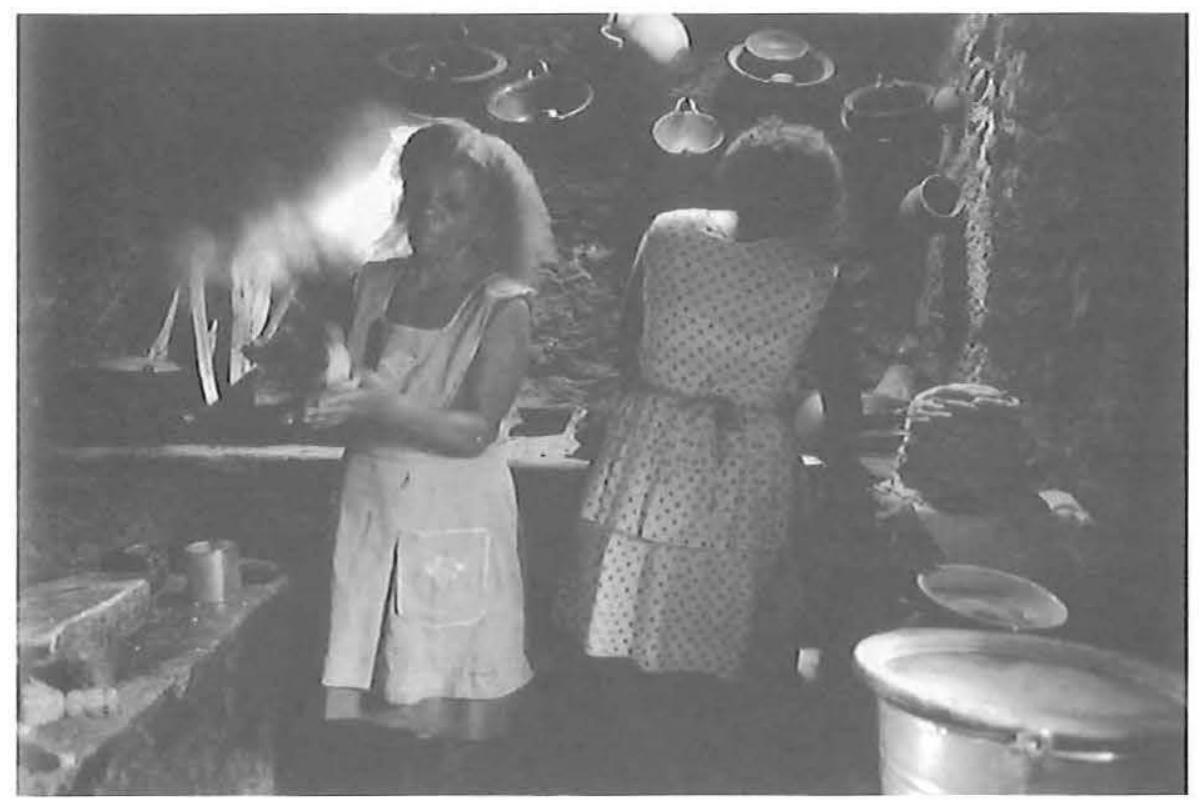

FIG. 1 - Cuisine de doña Mencha, Sud-Veracruz (cliché de Manuel González).

prouvé que cette somme d'éléments distinctifs soit le fondement d'une altérité collectivement construite et assumée. Les identités régionales intègrent ces éléments et bien d'autres qui, eux, ne sont pas spécifiquement « d'origine africaine » : l'histoire partagée, le type de hiérarchie socio-économique, le rôle des élites, les alliances et rivalités interrégionales, l'environnement et les conditions matérielles de la production et reproduction sont autant de facteurs qui concourent à l'édification d'un "nous » régional, tout autant que les " particularités culturelles » si souvent mises en avant. Les études sur les identités régionales ont depuis longtemps démontré que le champ culturel n'est signifiant que s'il est collectivement réinterprété dans le cadre plus vaste des rapports sociaux, politiques et économiques à l'intérieur et vers l'extérieur de la région (de la Peña 1981 ; Lomnitz 1995).

En résumé, l'approche ethnographique actuelle a du mal à se défaire du biais " folklorisant » et en fin de compte essentialiste qui l'a justifiée à ses débuts, lorsqu'il lui fallait " prouver » la présence et la pertinence des " particularités » noires ou d'origine africaine. En assimilant l'identité au seul champ culturel, elle «fabrique» de l'identité à partir de pratiques culturelles (voir les «cultures identitaires » d'Agier 2001) et risque de mener à une vision simplifiée et fragmentée de sociétés régionales beaucoup plus complexes. 
Certaines recherches récentes s'inscrivent cependant dans des approches plus contemporaines. En situant sa réflexion dans le débat sur l'identité nationale et les mythes de construction identitaire, Laura Lewis (2000) décentre la problématique afromexicaine et démonte les mécanismes d'appartenance et d'identification multiple et souvent contradictoire, dans le cas de la Costa Chica de Oaxaca. Elle montre ainsi la voie d'une autre anthropologie possible, aux côtés de chercheurs qui, dans la même région, abordent la question à l'occasion de l'analyse de la parenté (Díaz Pérez 2003), des systèmes de production (Quiroz Malca 1998), de la construction historique régionale (Pépin Lehalleur 2003; Delgado Calderón 2000) ou des dispositifs politiques (Lara Millán 2003). Sans a priori ni recherche d'ethnicité labellisée, ces auteurs intègrent la problématique de la différence dans un questionnement plus large sur les sociétés régionales et les dynamiques sociales. Ils rejoignent alors les orientations déjà mentionnées à propos des travaux historiques dans le Veracruz et qui ont fait leurs preuves dans d'autres régions afroaméricaines (pour la Colombie, voir Losonczy 2002). Ce ne sont ni l'ethnicité ni l'appartenance à un "groupe ethnique » qui ordonnent la vie sociale, mais plutôt des pratiques qui intègrent les différences identitaires à divers niveaux et dans des configurations variables selon les arènes et les enjeux (de la parenté, du rituel, de la production, du jeu politique, etc.). Bien présent dans la vie quotidienne individuelle et collective, le paramètre identitaire « noir » dessine des contrastes ou des voisinages sans pour autant composer de barrière et de frontière entre groupes et offre donc peu de prise à une conceptualisation « multiculturelle » et « interethnique » de la société. C'est pourtant bien ainsi que cherchent à la présenter certains courants qui se développent désormais rapidement dans le domaine culturel, social ou politique.

\section{LES PORTEURS DE L'ETHNICISATION}

Le rôle des institutions ${ }^{23}$ est décisif dans le biais ethnographique particularisant. Le programme «La troisième racine", qui a suscité de nombreuses monographies, trouve sa raison d'être dans la reconnaissance, puis la diffusion des traits culturels " spécifiques » et "distinctifs » des groupes de population noire et morena. Comme s'il fallait récupérer des siècles de déni en quelques années, on veut désormais prouver l'existence et la richesse d'une culture autre, en décrivant ses particularités dans plusieurs domaines, musique, danse, carnaval, religiosité, gestuelle ou tradition orale, c'est-à-dire les champs du folklore où les traditions locales sont objectivement « différentes » et susceptibles de description. Ainsi la musique jarocha dans le Veracruz se redécouvre " noire », et même africaine, de même que le son de artesa sur la Costa Chica, le carnaval de Coyolillo et même le zapateado du Michoacan, proche de celui du Jalisco. Les événements et les produits culturels (festivals, fêtes, conférences, vidéos, cédéroms ${ }^{24}$ ) aflìchent désormais " l'Afrique » au programme et participent à des processus de 
reconstruction et d'invention identitaires parfois stupéfiants par leur rapidité et leur succès. Ainsi les villages de Coyolillo (Martínez Maranto 1994) et Yanga (Cruz Carretero et al. 1989), dans le Veracruz, sont-ils désormais présentés comme "noirs ", chose difficilement concevable il y a à peine quinze ans. La récupération de l'histoire par les chercheurs, l'organisation d'ateliers de danse et de musique animés par des artistes, le tenue de séminaires scientifiques ou encore l'invitation d'activistes et sympathisants noirs étrangers, sont autant d'instruments de cette invention de la tradition, dont l'opérationalité repose sur le financement des uns ou des autres par les institutions culturelles et académiques.

Les institutions culturelles mexicaines disposent ainsi depuis plusieurs années d'instruments et de canaux efficaces pour divulguer l'idée d'une culture noire, rapidement interprétée comme l'expression d'une " identité noire ». L'accueil des populations concernées vis-à-vis de ces nouvelles propositions identitaires oscille de la réticence à l'adhésion en fonction des contextes locaux, mais ne suscite en général ni enthousiasme ni rejet. Il dépend plutôt des formes concrètes qu'adopte l'action culturelle, selon qu'elle répond plus ou moins bien aux attentes locales (la musique a toujours beaucoup de succès par exemple, de même que les danses) et qu'elle apporte éventuellement des avantages tant matériels (accès à des bourses, financements) qu'immatériels (rencontres avec des étrangers, prestige régional, animation). Le carnaval de Coyolillo ou celui de Yanga (Veracruz), aujourd'hui présentés comme "noirs » et même " africains », seraient ainsi le résultat d'une véritable "inoculation identitaire », selon les propres mots de Sagrario Cruz (communication personnelle), anthropologue chargée à l'époque de ces actions culturelles à la Dirección General de Culturas Populares.

Du côté des chercheurs impliqués, l'affìchage "ethnique noir » ouvre des portes vers le monde noir nord-américain qui se presse auprès de ses « frères oubliés » du Mexique. Plusieurs anthropologues de ce pays voisin sont en train de faire leurs thèses de doctorat sur le thème de l'identité noire mexicaine (Vaughn 2004), et les universités proposent des programmes et des collaborations, que ce soit au Mexique (Xalapa en mars 2004) ou aux États-Unis (par exemple les universités de Floride, de Californie, de Howard). En général, ils se situent dans le registre de la lutte contre l'invisibilité historique des noirs au Mexique et le racisme que celle-ci traduirait, et plusieurs adoptent des positions franchement afrocentristes (Fauvelle-Aymar et al. 2000), allant jusqu'à soutenir l'impossible thèse de la présence de noirs en Amérique avant la conquête espagnole ${ }^{25}$. Ces chercheurs arrivent avec des discours bien construits et légitimés dans leurs milieux d'origine par des institutions universitaires consolidées, des financements ou des charges de cours. Ils introduisent des concepts comme par exemple ceux de racisme institutionnel ou diaspora afroaméricaine, qui ne sont pas forcément les plus adaptés à la situation mexicaine telle que nous l'avons ébauchée plus haut. Les rencontres avec les chercheurs mexicains qui ne manient pas ces appareils théoriques sont d'autant plus délicates qu'elles se réalisent dans des situations 
d'inégalité objective des conditions de la recherche dans l'un et l'autre pays. On peut se demander si ces universitaires nord-américains, souvent porteurs des théories post-coloniales et subalternes, ne reproduisent pas au Mexique les mécanismes d'imposition théorique qu'ils critiquent aux États-Unis. La même relation ambiguë, faite de malentendus tus ou sous-estimés, caractérise les rapports qui s'établissent dans le champ de l'activisme afroaméricain. En témoigne la réaction violente d'un leader noir international invité, lorsqu'il assista à une rencontre annuelle de " Mexico noir ", une des rares organisations prônant la conscientisation ethnique des populations afromexicaines. Il avait en effet du mal à se reconnaître dans ce public bigarré, et son discours sur la diaspora noire latinoaméricaine ne réveillait que peu d'échos parmi ses auditeurs, habitants de la Costa Chica et ruraux pour la plupart. Il en déduisit hâtivement l'absence " d'authenticité noire » et donc la non-pertinence de sa présence parmi eux.

Cependant, dans le même temps, se développent d'autres dynamiques ethnicisantes qui, elles, s'ancrent dans les réalités quotidiennes de certains de ses promoteurs. Ainsi le Musée des cultures afrométisses, à Cuajinicuilapa (Costa Chica de Guerrero, inauguré en 1999), résulte en grande partie d'une initiative de l'élite locale qui voulait créer un espace culturel " de la communauté », sans y adjoindre de référence ethnique particulière ${ }^{26}$. Ils participaient ainsi à la vague de création de musées communautaires et écomusées encouragée par les institutions, en particulier l'INAH et la Dirección General de Culturas Populares, depuis la fin des années 1980 dans tout le pays. À la recherche de fonds et d'appuis institutionnels, ils rencontrèrent des interlocuteurs universitaires et politiques qui étaient, eux, intéressés par la problématique afro et offraient un matériel muséographique déjà élaboré sur ce thème. L'orientation " afrométisse » de ce musée - le seul de son genre au Mexique - s'est donc construite dans l'interaction et, largement, la coïncidence, mais son appropriation s'est faite sans heurt et assez rapidement de la part d'une population métisse qui ne voyait pas d'inconvénient majeur à célébrer ce volet de leur patrimoine culturel. D'autres initiatives ont vu le jour dans la même région, comme AFRICA (Alianza Fortalecimiento de las Regiones Indigenas y Comunidades Afromestizas), une association créée et soutenue par un groupe d'instituteurs et intellectuels locaux qui s'intéressent à la culture régionale et à ses influences noires et indiennes. Pour sa part, l'association rapidement mentionnée ci-dessus, México Negro, est l'œuvre d'un prêtre catholique noir originaire de Trinidad, venu au Mexique pour organiser les populations noires déshéritées. Mais, après quinze ans de travail, il constate à chaque réunion annuelle la difficulté de mobiliser les foules sur des thématiques « afro ». Par son activisme social et ses réseaux personnels, il a en revanche réussi à se constituer en protagoniste incontournable des institutions en demande d'interlocuteurs organisés (pour la production, les programmes éducatifs ou de santé, l'animation culturelle). Il jouit dans ces milieux d'une légitimité certaine, qui lui permet de rendre visible la problématique « noire » bien au-delà des frontières régionales et 
d'attirer des militants noirs étrangers qui diffusent à leur tour, notamment sur le web, l'idée de l'émergence d'une " conscience noire » au Mexique. Dans la même logique de visibilité internationale, México Negro participe à certains réseaux afroaméricains (Afroamerica XXI), sans que cette adhésion n'ait pour l'instant de répercussion notable au niveau local.

Un quatrième groupe d'acteurs - après les institutions culturelles, les universitaires et le secteur associatif - se situe dans la sphère politique locale qui commence timidement à intégrer la dimension afro dans ses activités. Lors de son mandat 2001-2004, le maire de Tututepec (Oaxaca) prône ainsi l'interculturalité au niveau local avec l'organisation d'échanges scolaires et de manifestations sportives entre les «villages indiens » et les « communautés noires » (c'est l'un des rares à employer ce terme) qui cohabitent sur le territoire municipal. À Pinotepa Nacional, le comité municipal d'organisation des fêtes a décidé d'inclure dans le défilé de la fète nationale, pour la première fois en 2003, une " reine afrométisse» aux côtés des traditionnelles reines de l'Espagne et de l'Amérique indienne. Plus récemment et en vue des élections (du gouverneur de l'État en juillet et municipales en septembre 2004), des courants politiques de gauche, sur la côte de Oaxaca, organisèrent des réunions destinées, séparément, aux populations afrométisses (en octobre 2003) et indiennes (quelques semaines plus tard). Ces quelques initiatives redessinent l'espace public en légitimant une participation différenciée des groupes ethniques, dont désormais les afrométis, mais ne concernent à ma connaissance que la seule région de la Costa Chica de Oaxaca.

\section{CONCLUSION : LES PISTES DE RECHERCHE}

L'absence de catégorisation externe de la part de l'État mexicain vis-à-vis des populations afrométisses n'a pas fourni de cadres stables de dialogue et de confrontation identitaire par lesquels « un groupe afrométis » aurait pu se construire dans le temps. En corollaire, elle s'est accompagnée d'une certaine liberté pour inventer d'autres formes d'identification et de positionnement dans les sociétés locales et régionales.

Un premier travail consisterait dès lors à repérer la diversité des expressions identitaires (politique dans telle région, culturelle dans telle autre, ethnique ailleurs) et à en expliquer les ressorts aux diverses échelles, dans divers contextes géographiques. L'intérêt immédiat de cette ligne de recherche serait de démontrer l'inconsistance des positions ethnicisantes qui concourent aujourd'hui à une ethnogenèse stéréotypée des " afromexicains ». L'autre intérêt, à plus long terme, serait de comprendre en quoi l'espace intervient dans la construction des identités en participant à des rapports de pouvoir entre acteurs et entre niveaux d'action. Il est particulièrement important de combiner les échelles d'analyse pour ne pas juxtaposer les monographies mais au contraire repérer les modes d'interconne- 
xion des espaces qui, comme le suggèrent Gupta et Ferguson (1997), façonnent les identités dans le temps. Dans le cas des études localisées, « au lieu d'assumer l'autonomie des communautés primordiales, il nous faut examiner la façon dont se forme la communauté à partir d'un espace interconnecté qui a toujours existé » (ibid., p. 36. Traduction et italique O. H.) ${ }^{27}$. Après avoir montré que les logiques d'identification n'étaient pas les mêmes au niveau interpersonnel, au niveau collectif régional ou international, il nous faudra comprendre les mécanismes d'influence réciproque entre les niveaux et, surtout, ne pas les isoler les uns des autres.

La prise en compte de l'espace permet de décrire et d'analyser des configurations identitaires originales, que les habitants interprètent et re-signifient selon leurs propres intérêts et leurs possibilités du moment. Au sein même de la Costa Chica, par exemple, les modèles d'identification varient d'une localité à l'autre. Dans le bourg où les représentants coloniaux puis nationaux ont imposé un modèle de castes depuis des siècles, « les noirs » se perçoivent comme distincts des indiens et des blancs. À 50 kilomètres de là, l'absence historique de classe dirigeante blanche a permis le développement d'une micro société noire et afrométisse qui se conçoit dans l'altérité aux indiens voisins, sans que l'opposition noir/blanc n'apparaisse pertinente. Un peu plus loin encore, ces diverses catégories sont ignorées au profit d'une image idéalisée du "métis " qui intègre les différences sans les resignifier en termes ethniques (Hoffmann 2004). Ces dispositifs identitaires reposent sur des fondements économiques et politiques bien précis, où les rapports de domination et de hiérarchie ont donné du sens à certaines « différences » pour en ignorer d'autres. L'élite blanche du bourg a entretenu la catégorisation différenciée des noirs et des indiens pour se reproduire en tant que classe dominante sur les plans économique et politique, alors que, dans les autres cas, les rapports de domination se sont établis au sein des groupes subordonnés, ou à leur voisinage avec les autres subordonnés. La catégorisation ethnique était ici beaucoup moins opérationnelle.

Ces situations contradictoires sont peut-être l'occasion de revisiter la notion de métissage, en évitant les théories idéologisées et globalisantes pour s'intéresser aux pratiques locales. Explorant les multiples facettes des identités (collective ou personnelle, assignée ou réinventée, nationale ou ethnique, etc.), Wieviorka $(2004$, p. 11) précise qu'« il faut non seulement admettre qu'il y a là des enjeux et des problèmes distincts, mais, en plus, reconnaître qu'en pratique, souvent, ils se chevauchent et se mêlent pour ne jamais correspondre à des types qui seraient sociologiquement purs [...]. Le débat n'a rien à gagner à tout confondre, ou à emprunter à ce qui relève d'un type de problème les catégories permettant d'en aborder d'autres. C'est pourquoi, tout en pouvant évidemment apporter un éclairage utile, la philosophie politique ne saurait jamais se substituer à la connaissance concrète des situations réelles, historiques ». Une interprétation documentée et localisée des expériences de métissage pourrait ouvrir des voies, 
loin des impasses de l'universalisme et du particularisme. Toutefois, il faut bien reconnaître que la marge de manœuvre est étroite, avec un risque d'avortement précoce sous l'effet des courants radicaux, nord-américains et internationaux, qui prônent une catégorisation ethnicisante des sociétés nationales.

À une autre échelle, au niveau individuel, l'affïrmation d'une altérité « noire » est à la fois évidente et douloureuse, par le rappel constant de la différence dans des rapports sociaux non exempts de racisme. Aussi donne-t-elle lieu, comme dans beaucoup d'endroits de par le monde, à des stratégies d'évitement ou de reniement qui se traduisent par exemple dans le domaine linguistique. Les termes d'appellation varient beaucoup selon les contextes d'interlocution, et l'euphémisation semble croître avec la distance au lieu d'origine. On est « negro » en famille, " moreno » à la ville et « mexicain » face à l'étranger. Au niveau interpersonnel, il existe ainsi des espaces d'identification noire, mais aussi d'hybridation et de jeu identitaire qui contribuent à se situer par rapport à l'autre, et à transgresser la limite et la frontière vis-à-vis de l'autre. Ainsi conçue comme un instrument de positionnement, l'identification n'est pas stable ni figée, et pourrait participer à une éventuelle reformulation de la catégorie globale de « noir » ou " afrométis » en un sens plus fluide et interactif que les propositions "ethniques» actuelles. À ce sujet, Stuart Hall parle des identités culturelles comme étant «l'instable point d'identification ou point de suture, construit au sein des discours de l'histoire et de la culture. Non pas une essence mais un positionnement. Et il y a toujours des politiques de l'identité, des politiques du positionnement » (Hall 1994, p. 226. Traduction O. H.) ${ }^{28}$.

Dans cette conception de l'identité, les « traits culturels » sont autant d'outils de positionnement social et politique, manipulables par les individus et les groupes. Une " généalogie » de ces manipulations culturelles, par une approche historique, permettrait de comprendre l'évolution des groupes sociaux non pas en termes de reproduction, mais peut-être de stratégie de résistance, toujours dans l'interaction avec les sociétés voisines et englobantes. Pour rester dans le domaine linguistique, les afrométis de la Costa Chica ont développé une tradition orale d'une grande richesse, dont la particularité repose en partie sur le corpus linguistique utilisé. Comme dans la région du Pacifique colombien, les populations noires conservent en effet nombres d'expressions venant de l'espagnol « archaïque ${ }^{29}$. Dépositaires d'un capital linguistique oublié par d'autres, les afrométis l'utilisent aujourd'hui encore dans leurs productions culturelles (poésies, corridos) et dans le quotidien, par des expressions humoristiques ou ironiques qui ne sont comprises que par eux et qui pourraient faire penser à une forme de " marronnage linguistique ». Utilisant l'outil du maître - la langue imposée avec l'esclavage -, ils le détournent de son usage standard et en font un instrument de communication accessible à certains seulement, depuis l'intérieur. Si la piste commence à être explorée par des linguistes (Althoff 1994 ; Githiora 1999), elle gagnerait à l'être également par des anthropologues. 
Les afromexicains contemporains se distinguent-ils par une « identité culturelle », une « identité ethnique » ou encore une " identité collective » ? L'objectif de l'article était de montrer qu'il n'est ni possible ni souhaitable de trancher sans tomber dans des simplismes qui obstruent la compréhension. Pour autant, les distinctions analytiques restent valides. En effet, selon les contextes, les espaces et les rapports locaux de subordination, les afromexicains activent certaines options plutôt que d'autres, individuellement ou collectivement. Les processus de construction identitaire sont pour l'instant encore très inégaux dans l'espace social et géographique, en grande partie à cause du (ou grâce au) désintérêt de l'État et des institutions pour un groupe très minoritaire et ne disposant pas de ressources stratégiques. C'est de l'extérieur des sociétés régionales et du pays que se dessinent le plus nettement les intentions de constituer un " groupe ethnique " noir mexicain qui pourrait rejoindre alors les «frères de la diaspora » latinoaméricaine. Ces tendances, si elles se confirmaient, risqueraient de gommer des processus autrement plus complexes, partagés entre le fait d'assumer un métissage ancien et celui de se faire enfin reconnaître comme différent. *

* Manuscrit reçu en mars 2005, accepté pour publication en juin 2005.

\section{NOTES}

1. Je remercie Anath Ariel de Vidas qui a bien voulu commenter une première version de ce texte et m'apporter d'intéressantes suggestions.

2. Comme d'autres catégories sociales (ouvriers, femmes, pauvres), les catégories ethniques sont des constructions aux frontières changeantes, que l'analyse ne saurait réduire à un groupe stable et facilement délimitable selon des critères « objectifs », comme c'est le cas des nationalités légalement constituées. C'est pourquoi je conserve les majuscules pour les nationalités (Mexicain), mais je n'utilise que des minuscules pour les ethnonymes (mixtèques, mames) ou les catégories ethniques (indiens, afrométis). Pour les mêmes raisons, j'adopte l'orthographe courante en Amérique latine (afromexicain au lieu d'Afro-Mexicain). Dans les deux cas, les substantifs utilisés se rapportent à des ensembles sociaux en redéfinition permanente, et non à des catégories légales ou sociales aux limites clairement établies.

3. "If anthropologists like it or not, it appears that people - and not only those with power - want culture, and they often want it precisely in the bounded, reified, essentialized and timeless fashion that most of us do now reject $"$.

4. "Central and culturally valued institutions and activities in an ethnic group may be deeply involved in its boundary maintenance by setting internal processes of convergence into motion 》.

5. Dans le cadre du projet IDYMOV, « Identidades y movilidades, una comparación MéxicoColombia ", CIESAS/IRD/ICANH, 2003-2006 (www.idymov.com).

6. La population « indigène " est estimée de $10,5 \%$ à $12,5 \%$ de la population totale du pays selon les critères utilisés. Est considéré comme « indigène » l'individu locuteur d'une langue indienne (critère le plus restrictif), membre d'une famille comprenant des locuteurs de langue indienne, ou encore personne se considérant membre d'une communauté indienne (auto-adscription, critère le plus large) (Serrano Carreto et al. 2002).

7. J'adopte le terme afromexicain ou afromexicaniste puisqu'il commence à faire consensus au Mexique, de la même façon qu'il s'est imposé dans d'autres pays d'Amérique latine (afrocolombien, 
afrobrésilien, etc.). Le terme d'afrodescendants est rarement utilisé et se réfère plus directement aux débats internationaux. En revanche, le terme " noir » ne fait aucun consensus, ni parmi les chercheurs, ni parmi les populations qui souvent ne se reconnaissent pas comme telles et utilisent des appellations régionales.

8. Y compris dans la version « multiculturelle » de la nation après la réforme constitutionnelle de 1992, par laquelle l'article 4 est modifié et énonce que la nation mexicaine a une composition pluriculturelle qui repose à l'origine sur ses « peuples » indiens.

9. Ce racisme ne se restreint évidemment pas au champ des interactions interpersonnelles et concerne la société dans son ensemble, aujourd'hui comme hier, par exemple lors de la Révolution mexicaine qui refonda l'identité nationale sans pouvoir « régler » la question du racisme (Knight 1990).

10. Les rébellions de Yanga (début $\mathrm{xvII}^{\mathrm{e}}$ siècle) et, surtout, dans la région de Córdoba (Veracruz)en 1735 menèrent à la formation de palenques, "villages de noirs libres ». Le premier fut fondé en 1640 sous le nom de San Lorenzo de los Negros (aujourd'hui appelé Yanga), suivi, un siècle plus tard, par le village de Nuestra Señora de Amapa (Naveda Chávez-Hita 1987).

11. Lors du recensement de 1777 , un prêtre faisait part des diflicultés rencontrées pour préciser la " caste » des personnes et ajoutait que, dans sa paroisse, " il n'a pas été établi de recensement séparé pour les seuls Espagnols, un autre pour les métis, un autre pour les mulâtres et un autre pour les indiens parce que toutes les castes habitent dans la ville, et dans une maison il est courant de rencontrer toutes les "qualités", et même dans une même famille on voit que le mari est d'une qualité, la femme d'une autre et les enfants d'une autre encore » (cité par Sánchez Santiró 2003, p. 41).

12. C'est-à-dire bien avant les émancipations en France (dont la définitive date de 1848), en Colombie (1851), aux États-Unis (1865), à Cuba (1886) et au Brésil (1888).

13. Certains clichés ont la vie longue mais ne correspondent pas aux évidences historiques. L'un d'eux consiste à penser à une répartition stricte de la présence noire au Mexique, qui aurait été réduite aux seules côtes atlantique (Veracruz-Tabasco) et pacifique (Costa Chica de Guerrero et Oaxaca). Comme on l'a dit, la population noire était initialement présente sur l'ensemble du territoire national, et certaines régions traditionnellement qualifiées de " blanches » ou " créoles », comme le Jalisco ou le nord du pays, ont connu de très importantes populations noires au XvI ${ }^{c}$ siècle (Becerra 2002 ; Nájera 2002), aujourd'hui disparues et effectivement « diluées » par métissage.

14. Il s'agit donc ici de catégories d'usage, comme on le verra dans la suite du texte.

15. «On ne peut nier que dans l'hybridation le facteur noir fut prépondérant et que, pour cette raison, le métis atuel de Cuajinicuilapa est principalement noir, c'est-à-dire, un afro-métis » (Aguirre Beltrán 1989, p. 65. Traduction O. H.).

16. En introduction à son ouvrage ethnographique, Aguirre Beltrán (1989, p. 7. Cet auteur utilise les minuscules pour les catégories identitaires - indiens, noirs, mixtèques -, suivant l'usage généralisé en castillan) réaffirme le cas exceptionnel de la Costa Chica en rappelant qu'au Mexique « le noir n'existe plus en tant que groupe différencié ».

17. Avec, en particulier, la création de l'Institut national indigéniste (INI) en 1948.

18. Notamment dans les États de Guanajuato et Veracruz.

19. Centro de Estudios sobre Asia y África.

20. Groupe de recherches sur les Caraibes.

21. Ce ne sont que quelques références indicatives, le corpus étant désormais trop imposant pour citer exhaustivement les auteurs ; voir les bibliographies mentionnées plus loin.

22. Aguirre Beltrán (1989) mentionne souvent «l'ethos violent » des noirs de la Costa Chica.

23. Que ce soit au niveau fédéral ou dans les États, avec par exemple la Dirección General de Culturas Populares, le Consejo Nacional para la Cultura y las Artes, l'Instituto Veracruzano de Cultura ou l'Instituto Oaxaqueño de Cultura.

24. Il n'est pas possible d'énumérer ici tous les festivals ou événements culturels de ce type. Mentionnons seulement le festival annuel afrocaribéen de Veracruz créé à la fin des années 1980, le séminaire « África en México » célébré en 2004 à Xalapa, ou les manifestations de " danse et musique 
noires " à Mexico et en province. Ils ont tous en commun de remettre à l'honneur la culture afromexicaine en insistant sur ses « racines » africaines.

25. Ces thèses sont clairement et fermement réfutées par les archéologues depuis plusieurs années. Elles persistent pourtant, comme par exemple lors du séminaire international co-organisé par l'université de Californie de Santa Barbara et intitulé « L'Afrique au Mexique », à Xalapa en mars 2004, où plusieurs archéologues mexicains ont soutenu ces orientations (Casimir de Brizuela 2004; Cuevas 2004). Ce qui, aux yeux des professionnels, constitue un véritable " retour au $\mathrm{xix}^{\mathrm{e}}$ siècle » est d'autant plus préoccupant qu'il ne suscite pas, pour l'instant, de réactions fortes dans le milieu universitaire mexicain - sauf peut-être dans le cercle restreint des spécialistes - qui préfère ignorer les quelques déviants - certes très minoritaires - et ne pas engager de polémiques à ce sujet. L'évolution constatée aux États-Unis, où ces thèses sont désormais enseignées dans certaines universités, devrait nous alerter sur la nécessité de contrer publiquement ces discours militants présentés comme "s scientifiques ».

26. Entretiens réalisés à Cuajinicuilapa (Guerrero) en janvier 2003, auprès des promoteurs du musée à l'époque de sa création, des responsables actuels du musée ainsi que des autorités politiques et culturelles de la ville aux deux périodes.

27. "Instead of assuming the autonomy of the primeval community, we need to examine how it was formed as a community out of the interconnected space that always existed ".

28. "Cultural identities (which) are the point of identification, the unstable point of identification or suture, which are made within the discourses of history and culture. Not an essence but a positionning. Hence, there is always a politics of identity, a politics of position ".

29. À la fin du XIXe siècle, un observateur précisait que les habitants de Cuajinicuilapa «sont de race africaine » et que, pour cette raison, ils « parlent l'espagnol ancien » (cité par Aguirre Beltrán 1989, p. 63).

\section{RÉFÉRENCES BIBLIOGRAPHIQUES}

\section{AGIER Michel}

2001 "Le temps des cultures identitaires. Enquête sur le retour du diable à Tumaco (Pacifique colombien) ", L'Homme, 157, pp. 87-114.

\section{Agudelo Carlos Efren}

1999 "Participation politique des populations noires en Colombie », Cahier des Amériques latines, 30, pp. 151-176.

\section{Aguirre Beltrán Gonzalo}

1972 La población negra de México : estudio ethnohistórico, Fondo de Cultura Económica, Mexico [1946].

1989 Cuijla : esbozo etnográfico de un pueblo negro, Fondo de Cultura Económica, Mexico [1958].

Alberro Solange B. de

1988 "Negros y mulatos : la integración dolorosa », Inquisición y sociedad en México, 1571-1700, Fondo de Cultura Económica, Mexico, pp. 455-487.

\section{Alcántara López Álvaro}

2002 "Negros y afromestizos del puerto de Veracruz. Impresiones de lo popular durante los siglos XVII y XVIII ", in Bernardo García Díaz et Sergio Guerra Vilaboy, éds, La Habana/Veracruz, Veracruz/La Habana. Las dos orillas, Universidad Veracruzana, Mexico, pp. 166-175. 


\section{Althoff Daniel}

1994 "AfroMestizo speech from Costa Chica, Guerrero : from Cuaji to Cuijla 》, Language Problems and Language Planning, 18 (3), pp. 242-256.

\section{Argyriadis Kali et Stefania CAPONE}

2004 "Cubanía et santeria. Les enjeux politiques de la transnationalisation religieuse (La Havane-Miami) », Civilisations, LI (1-2), pp. 81-137.

Arocha Jaime

1999 Ombligados de Ananse. Hilos ancestrales y modernos en el pacifico colombiano, Universidad Nacional de Colombia/CES, Bogota.

Barabas Alicia M. et Miguel A. Bartolomé

1986 Etmicidad y pluralismo cultural : la dinámica étnica en Oaxaca, CNCA, Mexico, coll. « Regiones ».

BARTH Fredrik

1994 "Enduring and emerging issues in the analysis of ethnicity ", in Hans Vermeulen et Cora Govers, éds, The anthropology of ethnicity. Beyond " Ethnic groups and boundaries ", Het Spinhuis, Amsterdam, pp. 11-32.

1995 "Les groupes ethniques et leurs frontières", in Philippe Poutignat et Jocelyne Streiff-Fenart, éds, Théories de l'ethnicité, PUF, Paris, pp. 203-249 [1969].

\section{Becerra Celina}

2002 "Población africana en una sociedad ranchera », Estudios Jalisciences, 49, pp. 7-19.

Campos Luis Eugenio

1999 «Negros y morenos. La población afromexicana de la Costa Chica de Oaxaca ", in Alicia M. Barabas et Miguel A. Bartolomé, éds, Configuraciones étnicas en Oaxaca. Perspectivas etnográficas para las autonomías. II. Mesoetnias, CNCA/INAH/INI, Mexico, pp. 145-182.

Carroll Patrick J.

1991 Blacks in colonial Veracruz. Race, ethnicity and regional development, University of Texas Press, Austin.

Casimir de Brizuela Gladys

2004 "Hubo negros en América Precolombina. En busca de una metodología », 3th Anmual conference "África en México ", Xalapa, Veracruz, 22-24 de marzo 2004.

\section{Castañon González Guadalupe}

2002 Punición y rebeldia de los negros en la nueva España en los siglos XVI y XVII, Instituto Veracruzano de la Cultura, Mexico, coll. " Sextante».

\section{Castillo Gómez Amaranta Arcadia}

2000 El papel de los estereotipos en las relaciones interétnicas : mixtecos, mestizos y afromestizos en Pinotepa Nacional, Oaxaca, tesis de licenciatura en antropología social, ENAH, Mexico. 


\section{Cervántes Delgado Roberto}

1984 "La Costa Chica. Indios, negros y mestizos », in Margarita Nolasco, éd., Estratificación étnica y relaciones interétnicas, ENAH, Mexico, pp. 37-50, coll. «Científica » 135.

\section{Chávez Carvajal María Guadalupe}

1995 "Negros y mulatos en Michoacán », in Agustín Jacinto Zavala et Álvaro Ochoa Serrano, éds, Tradición e identidad, El Colegio de Michoacán, Zamora, pp. 396-397.

\section{Cruz Sagrario}

1989 Identidad en una comunidad afromestiza del centro de Veracruz : la población de Mata Clara, tesis de licenciatura, Fundación Universidad de las Américas, Puebla.

\section{Cruz Carretero Sagrario et al.}

1989 El Carnaval en Yanga : notas y comentarios sobre una fiesta de la negritud, Consejo Nacional para la Cultura y las Artes, Mexico.

Cuevas Fernández Hector

2004 " ¿ Presencia negra en el México arqueológico ?», 3th Annual conference "África en México », Xalapa, Veracruz, 22-24 de marzo 2004.

CUNin Elisabeth

2004 Métissage et multiculturalisme en Colombie. Le " noir » entre apparences et appartenances, L'Harmattan/IRD, Paris, coll. "Connaissance des Hommes ».

DE LA PEÑa Guillermo

1981 «Los estudios regionales y la antropología social en México », Relaciones. Estudios de Historia y Sociedad, I (8), pp. 43-93.

Delgado Calderón Alfredo

2000 «La conformación de regiones en el Sotavento veracruzano : una aproximación histórica ", in Léonard Eric et Emilia Velázquez, éds, El Sotavento veracruzano. Procesos sociales y dinámicas territoriales, CIESAS/IRD, Mexico, pp. 27-42.

Díaz Pérez María Cristina

1994 Descripción etnográfica de las relaciones de parentesco en tres comunidades afromestizas de la Costa Chica de Guerrero, tesis de licenciatura de etnología, ENAH, Mexico.

2003 Queridato, matrifocalidad y crianza en tres comunidades de la Costa Chica, CNCA/Unidad Regional Guerrero/PACMYC, Mexico.

Fauvelle-Aymar François-Xavier, Jean-Pierre Chrétien et Claude-Hélène Perrot

2000 Afrocentrismes. L'histoire des Africains entre Égypte et Amérique, Karthala, Paris.

FLANET Véronique

1977 Viviré si Dios quiere. Un estudio de la violencia en la Mixteca de la costa, INI, Mexico, coll. " Antropología Social » 55. 
García de León Antonio

1992 "El Caribe afroandaluz : permanencias de una civilización popular », $L a$ Jornada semanal, domingo 12 de enero 1992, pp. 27-33, Mexico.

1993 «Comunidad histórica e identidad regional : los casos de Chiapas y Veracruz ", in Ricardo Ávila et Thomas Calvo, éds, Identidades, nacionalismos y regiones, Universidad de Guadalajara/Universidad Complutense de Madrid, Mexico, pp. 187-197.

Githiora Chege John

1999 Lexical variation in discourse : socio-racial terms and identity in an afromexican community, $\mathrm{PhD}$, department of linguistics and languages, Michigan State University, East Lansing.

Glazer Nathan et Daniel MoyniHaN

1975 Ethmicity : theory and experience, Harvard University Press, Cambridge.

GofFman Erving

1991 Les cadres de l'expérience, Les Éditions de Minuit, Paris [1974].

GRUZINSKı Serge

1999 La pensée métisse, Fayard, Paris.

Guevara Sanginés María

1994 «Participación de los africanos en el desarrollo del Guanajuato colonial », in Luz María Martínez Montiel, éd., Presencia africana en México, CNCA, Mexico, pp. 133-198.

Gupta Akhil et James Ferguson

1997 "Beyond "culture" : space, identity, and the politics of difference », in Akhil Gupta et James Ferguson, éds, Culture, power; place explorations in critical anthropology, Duke University Press, Durham/Londres, pp. 33-51.

Gutiérrez Ávila Miguel Ángel

1988 Corrido y violencia : entre los afromestizos de la Costa Chica de Guerrero y Oaxaca, Universidad Autónoma de Guerrero, Mexico.

HaLl Stuart

1994 "Cultural identity and diaspora ", Anthro 251. Issues on Cultural Studies, winter, Bookstore, Stanford, pp. 222-237.

Herrera Casasús María Luisa

1994 "Raíces africanas en la población de Tamaulipas », in Luz María Martínez Montiel, éd., Presencia africana en México, CNCA, Mexico, pp. 463-523.

HoFFManN Odile

2001 «Flux et reflux des catégories identitaires. Une lecture des politiques foncières au Mexique et en Colombie ", in Marie-France Prévôt Schapira et Hélène Rivière d'Arc, éds, Les territoires de l'État-Nation en Amérique latine, IHEAL Éditions, Paris, pp. 101-120.

2004 «De las "tres razas" al mestizaje : diversidad de las representaciones colectivas acerca de "lo negro" en México », II Seminario Internacional IDYMOV, Bogotá, 8-11 de noviembre 2004, pp. 81-92, CIESAS-Golfo, Xalapa (également sur www.idymov.com). 
KNIGHT Alan

1990 «Racism, revolution and indigenismo : Mexico, 1910-1940 », in Richard Graham, éd., The idea of race in Latin America, 1870-1940, University of Texas Press, Austin, pp. 71-112.

LaRa MiLlán Gloria

2003 Raices de color: Afromexicanos en la construcción ciudadana, Costa Chica, Oaxaca, tesis de maestría en desarrollo rural, Universidad Autónoma Metropolitana, Mexico.

Lévine Hal B.

1999 "Reconstructing ethnicity ", Journal of the Royal Anthropological Institute, 5 (2), pp. 165-180.

Lewis Laura

2000 «Blacks, black Indians, Afromexicans : the dynamics of race, nation and identity in a Mexican moreno community (Guerrero) ", American Ethnologist, 27 (4), pp. 898-926.

LOMNITZ-Adler Claudio

1995 Las salidas del laberinto. Cultura e ideología en el espacio nacional mexicano, Joaquín Mortín-Planeta, Mexico.

Losonczy Anne-Marie

2002 «De cimarrones a colonos y contrabandistas : figuras de movilidad transfronteriza en la zona dibullera del Caribe colombiano ", in Claudia Mosquera, Mauricio Pardo et Odile Hoffimann, éds, Afrodescendientes en las Américas. Trayectorias sociales e identitarias, Universidad Nacional/ ICANH/IRD/ILSA, Bogotá, pp. 215-244.

Martínez Maranto Alfredo

1994 «Dios pinta como quiere : identidad y cultura en un pueblo afromestizo de Veracruz », in Luz María Martínez Montiel, éd., Presencia africana en México, CNCA, Mexico, pp. 525-573.

Martínez Montiel Luz María

1993a "La pluralidad del mestizaje », in Luz María Martínez Montiel et Juan Carlos Reyes, éds, III Encuentro Nacional de Afromexicanistas, Colima, CNCA/Gobierno del estado de Colima, Colima, pp. 20-40.

1993b "La cultura africana : tercera raiz», in Guillermo Bonfil Batalla, éd., Simbiosis de culturas. Los inmigrantes y su cultura en México, FCE, Mexico, pp. 111-180.

1994 Presencia africana en México, Consejo Nacional para la Cultura y las Artes, Dirección General de Culturas Populares, Mexico, coll. « Claves de América latina ».

MOEDANO NAVARRo Gabriel

1992 «Bibliohemerografia histórica y etnohistórica sobre la población de origen africano en México ", in Emma Pérez-Rocha et Gabriel Moedano Navarro, éds, Aportaciones a la investigación de archivos del México colonial y a la 
bibliohemerografia afromexicanista, INAH, Mexico, pp. 37-64, coll. « Fuentes », Serie Bibliografías.

1997 «Los afromestizos y su contribución a la identidad cultural en el pacifico sur : el caso de la tradición oral en la Costa Chica ", in Gabriel Moedano Navarro, éd., Pacífico Sur :¿ Una región cultural ?, CNCA, Mexico, pp. 1-7.

NÁJERa Mario Alberto

2002 «Los afrojalisciences », Estudios Jalisciences, 49, agosto, pp. 20-32.

NAVEDA ChÁvez-Hita Adriana

1987 Esclavos negros en las haciendas azucareras de Córdoba, Veracruz, 1690-1830, Historias Veracruzanas, Xalapa.

NEFF Francoise

1986 "Reflexiones sobre la identidad del afromestizo de la Costa Chica de Guerrero », in Jesús Jáuregui et Ives Marie Gourio, éds, Palabras devueltas, INAH/IFAL/CEMCA, Mexico, pp. 71-74.

Palmer Colin Alphonsus

1993 "Afro-Mexican culture an consciousness during the Sixteenth and Seventeenth Centuries », in Joseph E. Harris, éd., Global dimensions of the African diaspora, Howard University Press, Washington, D. C., pp. 125-135.

Pépin Lehalleur Marielle

$2003 \quad$ « ¿ Existe el regionalismo popular? Reflexiones a partir de una región pluri-étnica », in Jaime Preciado, Hélène Rivière d'Arc, Luis Alfonso Ramírez et Marielle Pépin Lehalleur, éds, Territorios, actores y poder: Regionalismos emergentes en México, Universidad de Guadalajara/Universidad Autónoma de Yucatán, Guadalajara, pp. 25-48.

Quiroz Malca Haydee

1998 La mujeres y los hombres de la sal. Un proceso de producción y reproducción cultural en la Costa Chica de Guerrero, tesis de doctorado en antropología social, Universidad Iberoamericana, Mexico.

ReCONDo David

2001 "Mexique : multiculturalisme et démocratisation dans l'Oaxaca », Problèmes d'Amérique latine, 41, avril-juin, pp. 45-70.

SAHLINS Marshall

1999 "Two or three things I know about culture ", The Journal of Royal Anthropological Institute, 5 (3), pp. 399-422.

SÁNCHEZ SANTIRó Ernest

2003 Padrón del arzobispado de México 1777, AGN, Mexico.

Serrano Carreto Enrique, Arnulfo Embriz Osorio et Patricia Fernández Ham, éds 2002 Indicadores socioeconómicos de los pueblos indigenas de México, INIPNUD/CONAPO, Mexico.

Touraine Alain

1988 "Modernité et spécificités culturelles ", Revue internationale des Sciences Sociales, XL (4), pp. 497-511. 
VASCONCELOS José

1958 «La raza cósmica », in Obras completas. II, Libreros Mexicanos, Mexico, pp. 903-942.

VAUGHN Bobby

2004 Negros, una apreciación global breve, disponible sur www.mexconnect.com/ $\mathrm{mx} \_$/feature/ethnic/bv/costa.htm.

VeLASCo Baltazar

2002 «La Costa Chica », Revista Fandango, 2, verano, pp. 1-2.

Velázquez Gutiérez María Elisa

1994 «Mujeres afromexicanas en la Nueva España », ponencia presentada en el IV Encuentro de Afromexicanistas, Instituto Veracruzano de Cultura, Veracruz.

Velízquez María Elisa et Ethel Correa, éds

2005 Poblaciones y culturas de origen africano en México, INAH, Mexico, coll. "Africanía ».

WACHTEL Nathan

1992 "Note sur le problème des identités collectives dans les Andes méridionales ", L'Homme, 122-124, pp. 39-52.

Wieviorka Michel

2004 «Identités culturelles et démocratie », séminaire ERSIPAL/IHEAL, Paris. 